\title{
Frecuencia de disfunción olfatoria en mujeres embarazadas con infección por SARS-CoV-2
}

\author{
Sebastián Carranza-Lira, ${ }^{*}$ Maritza García-Espinosa² y Oscar Moreno-Álvarez ${ }^{3}$ \\ ${ }^{1}$ División de Investigación en Salud; '2Departamento Clínico de Complicaciones de la Segunda Mitad del Embarazo; ${ }^{3}$ Dirección General. Instituto \\ Mexicano del Seguro Social, Hospital de Gineco-Obstetricia 4 "Luis Castelazo Ayala", Ciudad de México, México
}

\section{Resumen}

Introducción: En pacientes con COVID-19 se ha reportado disfunción olfatoria y anosmia; en la mujer embarazada se presenta hasta en $\mathbf{2 4 . 2} \%$. Objetivo: Conocer la frecuencia con la que las mujeres embarazadas e infección por SARS-CoV-2 tienen disfunción olfatoria. Métodos: Se preguntó edad, edad gestacional, temperatura, presencia de constipación nasal o rinorrea, mialgias, cefalea, tos o dolor torácico, además de evaluar si las mujeres percibían e identificaban el aroma de jugo de uva, café en polvo y mentol. Se utilizaron medidas de tendencia central y dispersión, frecuencias y porcentajes. Se calculó sensibilidad, especificidad, valor predictivo positivo y negativo. La U de Mann-Whitney y el contraste de proporciones sirvieron para las comparaciones entre los grupos. Resultados: Hubo mayor proporción de mujeres con tos, cefalea, disnea, mialgias, odinofagia, rinorrea, dolor torácico y anosmia en mujeres positivas a SARS-CoV-2. De las pacientes sin COVID-19, 88.9 \% detectó cada uno de los aromas; solo $31.8 \%$ del grupo positivo detectó el aroma a uva, $47.7 \%$ el de café y $59.1 \%$ el de mentol, el cual tuvo los porcentajes más altos en sensibilidad (40\%), especificidad (21\%), valores predictivos positivo (59\%) y negativo (11\%). Conclusión: la disfunción olfatoria se presenta en un porcentaje importante de las mujeres embarazadas con COVID-19.

PALABRAS CLAVE: SARS-CoV-2. COVID-19. Disfunción olfatoria. Anosmia.

\section{Frequency of olfactory dysfunction in pregnant women with SARS-CoV-2 infection}

\begin{abstract}
Introduction: In patients with COVID-19, olfactory dysfunction and anosmia have been reported, which in pregnant women occur in up to $24.2 \%$. Objective: To know the frequency in which pregnant women with SARS-CoV-2 infection have olfactory dysfunction. Methods: Age, gestational age, temperature, presence of nasal constipation or rhinorrhea, myalgia, headache, cough or chest pain were asked. Whether patients perceived and identified the scent of grape juice, coffee powder and menthol was evaluated. Central tendency and dispersion measures, frequencies and percentages were used. Sensitivity, specificity, positive and negative predictive value were calculated. Mann-Whitney's U-test and contrast of proportions were used for comparisons between groups. Results: There was a higher proportion of women with cough, headache, dyspnea, myalgia, odynophagia, rhinorrhea, chest pain, and anosmia in SARS-CoV-2-positive women. In patients without COVID-19, $88.9 \%$ detected each one of the scents; only $31.8 \%$ of the positive group detected grapes scent, $47.7 \%$ coffee and $59.1 \%$ menthol, which had the highest percentages of sensitivity (40\%), specificity (21\%), positive predictive value (59\%) and negative predictive value (11\%). Conclusion: Olfactory dysfunction occurs in a significant percentage of pregnant women with COVID-19.
\end{abstract}

KEY WORDS: SARS-CoV-2. COVID-19. Olfactory dysfunction. Anosmia.

Correspondencia:

*Sebastián Carranza-Lira

E-mail: drsebastiancarranza@gmail.com
Fecha de recepción: 22-09-2020

Fecha de aceptación: 01-10-2020

DOI: $10.24875 / G M M .20000696$
Gac Med Mex. 2021;157:255-260

Disponible en PubMed

www.gacetamedicademexico.com

0016-3813/@ 2020 Academia Nacional de Medicina de México, A.C. Publicado por Permanyer. Este es un artículo open access bajo la licencia CC BY-NC-ND (http://creativecommons.org/licenses/by-nc-nd/4.0/). 


\section{Introducción}

El virus SARS-CoV-2 es el causante de la enfermedad COVID-19, la cual es transmisible de persona a persona y se asocia a distintos síntomas, entre los cuales la faringodinia y la congestión nasal son los más frecuentes en vías aéreas superiores, reportados en $12.4 \%$ y entre 3.7 y $88 \%$, respectivamente. ${ }^{1}$ Los síntomas más frecuentes son fiebre (85.6\%), tos $(68.7 \%)$, fatiga $(39.4 \%)$ y disnea $(21.4 \%){ }^{2} \sin$ embargo, también se ha encontrado disfunción olfatoria, cuya frecuencia oscila entre 34 y $68 \%{ }^{3}$

En un estudio en el que se analizaron 60 casos, $98 \%$ presentó disfunción olfatoria y $58 \%$, anosmia, ${ }^{3}$ mientras que en otro análisis se reportó que las alteraciones del olfato y del gusto se presentaron en 85.6 y $88 \% .^{1}$ En un estudio que comparó pacientes con COVID-19 positivos y negativos respecto a la pérdida de olfacción y gusto, se encontró que estos síntomas tuvieron mayor frecuencia en el primer grupo (68\% versus $16 \%$ y $71 \%$ versus $17 \%$, respectivamente). ${ }^{4}$ En un estudio de pacientes hospitalizados, se encontró que la frecuencia de alteraciones fue mayor en aquellos con enfermedad severa, aunque sin significación estadística (gusto 1.5 y $2.5 \%$, olfato 2.0 y $2.5 \%$ en pacientes con enfermedad severa y no severa, respectivamente). ${ }^{5}$

En $79.7 \%$ de las pacientes con obstrucción nasal o rinorrea se registró hiposmia o anosmia, que en $11.8 \%$ constituyó un síntoma temprano anterior a la aparición de otros. ${ }^{1,4,6}$

En mujeres embarazadas se reportó que la anosmia se presentó en $24.2 \%$ de las aquellas con COVID-19. ${ }^{7}$ Se ha reportado que en la mujer embarazada sin COVID-19 la frecuencia de hiposmia es de $7.1 \%$, mientras que en la no embarazada es de $2.9 \%{ }^{8}$

En pacientes con infección por SARS-CoV-2 se observó anosmia prolongada; 40 a $60 \%$ de los casos se recuperó espontáneamente, si bien solo $15 \%$ con anosmia y $25 \%$ con hiposmia recuperaron la olfacción normal. ${ }^{9}$

Con SARS-CoV-2 no se sabe cuál es el mecanismo por el que se afecta el epitelio olfatorio $\mathrm{y}$, por tanto, se afecta la olfacción; sin embargo, se ha planteado que existe daño al bulbo olfatorio y un proceso inflamatorio que temporalmente impide detectar los olores. ${ }^{9}$ También se ha planteado que están involucrados los receptores para la enzima convertidora de angiotensina 2 (ECA2) y la proteasa transmembrana de serina 2 en el epitelio olfatorio, necesarias para transferir los olores a las neuronas. ${ }^{10,11}$

Para la evaluación de la disfunción olfatoria existen varias pruebas, una de ellas es AROMA (Affordable Rapid Olfaction Measurement Array), que evalúa 14 aromas. Otras versiones más breves evalúan 12 ítems y otra, cuatro; está última con una sensibilidad de $91.2 \% .^{12}$

El objetivo del presente estudio fue conocer la frecuencia de disfunción olfatoria en las mujeres embarazadas con infección por SARS-CoV-2.

\section{Métodos}

Se trató de un estudio observacional, transversal, prospectivo, comparativo, abierto, controlado, en el que se estudiaron dos grupos de mujeres:

- Grupo I, mujeres embarazadas con infección por SARS-CoV-2 corroborada por PCR, evaluadas en la zona de triage o en hospitalización área COVID.

- Grupo II, mujeres embarazadas en quienes se sospechó COVID-19 y que fueron hospitalizadas; una vez internadas se realizó la prueba RT-PCR, la cual fue negativa para SARS-CoV-2.

Fueron incluidas consecutivamente las mujeres con prueba positiva para SARS-CoV-2 al momento de su evaluación al ingreso al hospital. En todas las mujeres se investigó el antecedente de diabetes, hipertensión crónica, asma, tabaquismo, neumopatía, cardiopatía, nefropatía e inmunodeficiencia secundaria a $\mathrm{VIH}$.

No fueron incluidas las mujeres con alteraciones nasales, antecedente de cirugía nasal, uso de medicamentos que pudieran modificar la olfacción (uso crónico de gotas nasales o sustancias inhaladas) 0 con COVID-19 grave, que no pudieran leer el consentimiento informado o a quienes no pudiera realizarse la prueba. Asimismo, no se incluyeron mujeres puérperas.

Después de que las mujeres firmaran el consentimiento informado, se registró edad (años), peso (kg), talla $(\mathrm{m})$, índice de masa corporal (IMC, $\mathrm{kg} /$ talla en $\mathrm{m}^{2}$ ); un IMC $\leq 25$ se consideró normal, > 25 a 30 indicó sobrepeso y $>30$, obesidad. Se investigó el número de gestas, partos, abortos, cesáreas y edad gestacional en el embarazo que se cursaba al momento de la investigación, así como presencia de tos, cefalea, disnea, mialgias, artralgias, odinofagia, constipación nasal o rinorrea, conjuntivitis, dolor torácico y anosmia. Se documentó temperatura $\left({ }^{\circ} \mathrm{C}\right)$, frecuencia cardiaca (normal $\leq 90$ latidos/minuto [lpm]), 
frecuencia respiratoria (normal $\leq 20$ respiraciones/ minuto [rpm]) y saturación de oxígeno (normal $\geq 90 \mathrm{~mm} \mathrm{Hg}$ ).

Posteriormente, a las mujeres se les proporcionó tres tubos Eppendorf, cada uno de los cuales contenía jugo de uva, café en polvo y mentol-alcanfor-eucalipto (Vick VapoRub ${ }^{\circledR}$, Vick México). Se les preguntó si percibían cada aroma y si los identificaban. Se consideró disfunción olfatoria a la incapacidad parcial o total para percibir correctamente los olores, que se evaluó a partir del número de identificaciones de aromas: ninguno, uno de tres, dos de tres, tres de tres.

Para el cálculo del tamaño de muestra se consideró un error tipo alfa de 0.01 y un error beta de 0.05 , con $60 \%$ de frecuencia de disfunción olfatoria en el grupo I y $5 \%$ en el grupo II (sin infección). Con base en una relación de 3:1 entre los grupos, se determinaron 43 y 15 pacientes para los grupos I y I| (MedCalc Statistical Software versión 18.5, MedCalc Software, Ostende, Bélgica; http://www.medcalc.org).

Se utilizaron medidas de tendencia central y dispersión, frecuencias y porcentajes. Para las comparaciones entre los grupos de las variables continuas se utilizó $U$ de Mann-Whitney y para las nominales, contraste de proporciones (chi cuadrada).

Se calculó sensibilidad, especificidad, valor predictivo positivo (VPP) y valor predictivo negativo (VPN). El proyecto fue autorizado por el Comité de Ética en Investigación y el Comité Local de Investigación en Salud, con el número de registro R-2020-3606-022; las pacientes proporcionaron su consentimiento para participar.

\section{Resultados}

Se estudiaron 62 pacientes, 44 en el grupo I y 18 en el grupo II. La mediana de la edad fue de 29 años (16-41) en el grupo I y de 30.5 años (22-43) en el grupo II. Al comparar el número de gestas, partos, abortos y cesáreas, así como las semanas de gestación, no existió diferencia entre los grupos (Tabla 1).

No hubo diferencia entre los grupos I y II respecto al peso: $75.5 \mathrm{~kg}$ (56-94) versus 77 (54- 113), $\mathrm{p}<0.207$; ni en cuanto a la talla: $1.59 \mathrm{~m}(1.47-1.74)$ versus $1.57 \mathrm{~m}$ (1.40-1.65), $p<0.207$; ni al IMC: 29.7 (21.9-36.7) versus 30.1 (21.1-44.1), $p<0.987$.

Al comparar ambos grupos se encontró que la proporción de mujeres con los siguientes síntomas fue significativamente mayor en el grupo I: tos, cefalea, disnea, mialgias, odinofagia, rinorrea, dolor torácico
Tabla 1. Edad y datos obstétricos en mujeres embarazadas positivas y negativas a infección por SARS-CoV-2

\begin{tabular}{|l|c|c|c|}
\hline Variable & $\begin{array}{c}\text { Positivas } \\
(\mathrm{n}=44)\end{array}$ & $\begin{array}{c}\text { Negativas } \\
(\mathrm{n}=18)\end{array}$ & $p$ \\
\hline Edad en años & $29(16-41)$ & $30.5(22-43)$ & 0.798 \\
\hline Semanas de gestación & $34(8-40)$ & $34.5(8-38)$ & 0.809 \\
\hline Gestas* & $2(1-6)$ & $2(1-6)$ & 0.832 \\
\hline Partos* $^{*}$ & $0(0-2)$ & $0(0-3)$ & 0.183 \\
\hline Abortos* $^{*}$ & $0(0-3)$ & $0(0-4)$ & 0.912 \\
\hline Cesáreas* $^{*}$ & $0(0-1)$ & $0(0-2)$ & 0.962 \\
\hline
\end{tabular}

*Los datos se presentan en media (mínima y máxima).

y anosmia. Los síntomas con mayor sensibilidad fueron la tos, la conjuntivitis y el dolor torácico, todos con $100 \%$; los que tuvieron mayor especificidad fueron la tos $(94 \%)$, la cefalea $(61 \%)$ y la anosmia $(61 \%)$. Los síntomas con mayor VPP fueron la tos (94\%), la fiebre $(64 \%)$ y la cefalea (48\%); y los síntomas con mayor VPN, la tos, la conjuntivitis y el dolor torácico, todos con $100 \%$ (Tabla 2).

La frecuencia cardiaca fue significativamente mayor en el grupo I al compararla con la del grupo II: 100 Ipm (70-151) versus 79 Ipm (60-197), $\mathrm{p}<0.008$. Respecto a la frecuencia respiratoria, no hubo diferencia significativa entre los grupos I y II: $20.5 \mathrm{rpm}$ (14-38) versus 22 rpm (14-23), p < 0.820. La saturación de oxígeno fue significativamente mayor en el grupo II en comparación con la del grupo I: $95.5 \%$ (78-98) versus $94 \%$ (66-98), $p<0.042$.

La proporción de mujeres con frecuencia cardiaca $>90$ Ipm fue superior en el grupo I en comparación con el grupo II: $59.1 \%(n=26)$ versus $16.7 \%(n=3)$, $p<0.004$. No hubo diferencia en la proporción de mujeres con frecuencia respiratoria $>20 \mathrm{rpm}$ en los grupos I y II: $50.0 \%(n=22)$ versus $61.1 \%(n=11)$, $p<0.576$. De igual forma, no la hubo en la proporción de mujeres con saturación de oxígeno < $90 \mathrm{~mm} \mathrm{Hg}$ : $15.9 \%(n=8)$ versus $5.6 \%(n=1), p<0.418$.

La frecuencia cardiaca tuvo sensibilidad de $78 \%$ y especificidad de $67 \%$; le siguió la saturación de oxígeno, con sensibilidad de $74 \%$ y especificidad de $53 \%$; y la frecuencia respiratoria, con sensibilidad de $45 \%$ y especificidad de $44 \%$. Del VPP, el valor mayor lo tuvo la frecuencia cardiaca (59\%), seguido por el de la frecuencia respiratoria (50\%) y el de la saturación de oxígeno (16\%). El VPN fue mayor para la saturación de oxígeno (94\%), seguido por el de la 
Tabla 2. Síntomas en mujeres embarazadas positivas y negativas a infección por SARS-CoV-2

\begin{tabular}{|c|c|c|c|c|c|c|c|c|c|}
\hline \multirow[t]{2}{*}{ Síntoma } & \multicolumn{2}{|c|}{$\begin{array}{l}\text { Positivas } \\
(n=44)\end{array}$} & \multicolumn{2}{|c|}{$\begin{array}{c}\text { Negativas } \\
(n=18)\end{array}$} & \multirow[t]{2}{*}{$\mathrm{p}$} & \multirow[t]{2}{*}{ Sensibilidad (\%) } & \multirow[t]{2}{*}{ Especificidad (\%) } & \multirow[t]{2}{*}{ VPP (\%) } & \multirow[t]{2}{*}{ VPN (\%) } \\
\hline & $n$ & $\%$ & $\mathrm{n}$ & $\%$ & & & & & \\
\hline Fiebre & 28 & 63.6 & 8 & 44.4 & 0.134 & 59 & 60 & 64 & 56 \\
\hline Tos & 34 & 77.3 & 8 & 44.4 & 0.018 & 100 & 94 & 94 & 100 \\
\hline Cefalea & 21 & 47.7 & 3 & 16.7 & 0.025 & 74 & 61 & 48 & 83 \\
\hline Disnea & 13 & 29.5 & 1 & 5.6 & 0.049 & 84 & 57 & 30 & 94 \\
\hline Mialgias & 16 & 36.4 & 1 & 5.6 & 0.014 & 87 & 60 & 36 & 94 \\
\hline Artralgias & 14 & 31.8 & 2 & 11.1 & 0.117 & 74 & 57 & 32 & 89 \\
\hline Odinofagia & 16 & 36.4 & 1 & 5.6 & 0.014 & 87 & 60 & 36 & 94 \\
\hline Rinorrea & 16 & 36.4 & 1 & 5.6 & 0.014 & 87 & 60 & 36 & 94 \\
\hline Conjuntivitis & 5 & 11.1 & 0 & 0 & 0.309 & 100 & 53 & 11 & 100 \\
\hline Dolor torácico & 14 & 31.8 & 0 & 0 & 0.006 & 100 & 59 & 32 & 100 \\
\hline Diarrea & 5 & 11.1 & 2 & 11.1 & 1.0 & 51 & 50 & 11 & 89 \\
\hline Anosmia & 19 & 43.2 & 2 & 11.1 & 0.019 & 80 & 61 & 43 & 89 \\
\hline
\end{tabular}

Tabla 3. Detección positiva de distintos aromas en mujeres embarazadas positivas y negativas a SARS-CoV-2

\begin{tabular}{|c|c|c|c|c|c|c|c|c|c|}
\hline \multirow[t]{2}{*}{ Aroma } & \multicolumn{2}{|c|}{$\begin{array}{l}\text { Positivas } \\
(n=44)\end{array}$} & \multicolumn{2}{|c|}{$\begin{array}{l}\text { Negativas } \\
(n=18)\end{array}$} & \multirow[t]{2}{*}{ p } & \multirow[t]{2}{*}{ Sensibilidad (\%) } & \multirow[t]{2}{*}{ Especificidad (\%) } & \multirow[t]{2}{*}{ VPP (\%) } & \multirow[t]{2}{*}{ VPN (\%) } \\
\hline & $\mathbf{n}$ & $\%$ & $\mathrm{n}$ & $\%$ & & & & & \\
\hline Uva & 14 & 31.8 & 16 & 88.9 & $<0.000$ & 26 & 14 & 32 & 11 \\
\hline Café & 21 & 47.7 & 16 & 88.9 & $<0.004$ & 35 & 18 & 48 & 11 \\
\hline Mentol & 26 & 59.1 & 16 & 88.9 & $<0.035$ & 40 & 21 & 59 & 11 \\
\hline
\end{tabular}

frecuencia cardiaca (83\%) y el de la frecuencia respiratoria (39\%).

Se encontró que $45.5 \%$ de mujeres del grupo I y $16.7 \%$ del grupo II alcanzaron 100 Ipm en la frecuencia cardiaca, punto de corte que tuvo sensibilidad de $73 \%$, especificidad de $60 \%$, VPP de $45.4 \%$ y VPN de $83.3 \%$.

En las pacientes sin COVID-19, $88.9 \%$ detectó de igual forma cada uno de los aromas; en el grupo I, $31.8 \%(n=14)$ detectó el aroma de uva, $47.7 \%$ $(n=21)$ el del café y $59.1 \%(n=26)$ el del mentol.

El aroma de uva tuvo la menor sensibilidad, especificidad, VPP y VPN; el café presentó valores intermedios y el mentol tuvo los porcentajes más altos en los cuatro parámetros: sensibilidad $40 \%$, especificidad $21 \%$, VPP $59 \%$ y VPN $11 \%$ (Tabla 3).
La detección de tres aromas fue menor en el grupo de mujeres con infección por SARS-CoV-2 en comparación con las mujeres sin infección: $31.8 \%(n=14)$ versus $88.9 \%(n=16), p<0.001$. En cuanto al número de aromas detectados, la identificación de tres tuvo sensibilidad de $26 \%$, especificidad de $21 \%$, VPP de $44 \%$ y VPN de $11 \%$ (Tabla 4 ).

\section{Discusión}

Es conocido que la infección por SARS-CoV-2 se asocia con disfunción olfatoria de distinto grado en 34 a $98 \%$ de los casos y que se presenta anosmia en 58 a $71 \%,{ }^{3}$ prevalencia mayor a la registrada en este estudio: de $40.9 \%$ en la mujeres del grupo I y de $11.1 \%(n=2)$ en el grupo grupo II, menor a $7.1 \%$ reportado. ${ }^{8}$ 
Tabla 4. Número de detecciones correctas de distintos aromas en mujeres embarazadas positivas y negativas a SARS-CoV-2

\begin{tabular}{|c|c|c|c|c|c|c|c|c|}
\hline \multirow[t]{2}{*}{$\begin{array}{l}\text { Número } \\
\text { detecciones }\end{array}$} & \multicolumn{2}{|c|}{$\begin{array}{c}\text { Positivas } \\
(n=44)\end{array}$} & \multicolumn{2}{|c|}{$\begin{array}{c}\text { Negativas } \\
(n=18)\end{array}$} & \multirow[t]{2}{*}{ Sensibilidad (\%) } & \multirow[t]{2}{*}{ Especificidad (\%) } & \multirow[t]{2}{*}{ VPP (\%) } & \multirow[t]{2}{*}{ VPN (\%) } \\
\hline & $\mathrm{n}$ & $\%$ & $\mathrm{n}$ & $\%$ & & & & \\
\hline Ninguna* & 18 & 40.9 & 2 & 11.1 & - & - & - & - \\
\hline 1 de 3 & 5 & 11.4 & 0 & 0 & 100 & 21 & 22 & 100 \\
\hline 2 de 3 & 7 & 15.9 & 0 & 0 & 100 & 21 & 28 & 100 \\
\hline 3 de $3^{*}$ & 14 & 31.8 & 16 & 88.9 & 26 & 21 & 44 & 11 \\
\hline
\end{tabular}

Respecto a los síntomas, se ha reportado que los más frecuentes son fiebre $(85.6 \%)$, tos $(68.7 \%)$, fatiga $(39.4 \%)$ y disnea $(21.4 \%){ }^{2}$ mientras que en este estudio fueron tos (63.6\%), cefalea (77.3), disnea $(47.7 \%)$ y mialgias $(29.5 \%) .^{13}$ En otro estudio se reportó que los síntomas basales en la totalidad de la población fueron fiebre (54\%), tos (66\%) y disnea (25\%); que en el grupo con enfermedad severa tuvieron una frecuencia de 93, 86 y $57 \%$, respectivamente. ${ }^{14}$

Los síntomas con mayor sensibilidad fueron la tos, la conjuntivitis y el dolor torácico, todos con $100 \%$; aquellos con mayor especificidad fueron la tos (94\%), la cefalea $(61 \%$ ) y la anosmia (61 \%). La tos $(94 \%)$, la fiebre $(64 \%)$ y la cefalea (48\%) constituyeron los síntomas con mayor VPP; la tos, la conjuntivitis y el dolor torácico representaron los síntomas con mayor VPN, todos con $100 \%$.

En otra investigación se documentó que la frecuencia respiratoria > $20 \mathrm{rpm}$ se presentó en $43 \%$ de los casos, la frecuencia cardiaca $>100 \mathrm{lpm}$ en $37 \%$ y la saturación de oxígeno $>95 \mathrm{~mm}-\mathrm{Hg}$ en $18 \%{ }^{14}$ en el presente estudio, la frecuencia cardiaca $>90 \mathrm{lpm}$ se observó en $59.1 \%$. Al considerar como punto de corte la frecuencia cardiaca de $100 \mathrm{lpm}$, esta se encontró en $45.5 \%$ de las mujeres embarazadas con COVID19 , semejante a lo reportado,,$^{14}$ y en $16.7 \%$ de la mujeres embarazadas sin infección por SARS-CoV-2, con una sensibilidad de $73 \%$, especificidad de $60 \%$, VPP de $45.4 \%$ y VPN de $83.3 \%$.

En este estudio se utilizaron solo tres aromas para evaluar la olfacción, esta prueba simplificada constituyó una fortaleza que permitió discriminar entre los grupos. De los tres aromas evaluados, el mentol obtuvo la mayor sensibilidad, especificidad, VPP y VPN (40, 21, 59 y $11 \%$, respectivamente).

Con base en lo anterior es posible concluir que la disfunción olfatoria se presenta en un porcentaje importante de las mujeres embarazadas con COVID-19.

\section{Conflicto de intereses}

Los autores declaran que no tienen conflicto de intereses.

\section{Financiamiento}

Los autores no recibieron patrocinio para llevar a cabo este artículo.

\section{Responsabilidades éticas}

Protección de personas y animales. Los autores declaran que para esta investigación no realizaron experimentos en seres humanos ni en animales.

Confidencialidad de los datos. Los autores declaran que siguieron los protocolos de su centro de trabajo sobre la publicación de datos de pacientes.

Derecho a la privacidad y consentimiento informado. Los autores obtuvieron el consentimiento informado de los pacientes o sujetos referidos en el artículo y que la documentación obra en poder del autor de correspondencia.

\section{Bibliografía}

1. Lechien JR, Chiesa-Estomba CM, de Siati DR, Horoi M, Le Bon SD, Rodríguez A, et al. Olfactory and gustatory dysfunctions as a clinical presentation of mild-to-moderate forms of the coronavirus disease (COVID-19): a multicenter European study. Eur Arch Otorhinolaryngol. 2020;277:2251-2261.

2. Lovato A, De Filippis C. Clinical presentation of COVID-19: a systematic review focusing on upper airway symptoms. Ear Nose Throat J Ear Nose Throat J. 2020:145561320920762.

3. Moein ST, Hashemian SMR, Mansourafshar B, Khorram-Tousi A, Tabarsi P, Doty RL. Smell dysfunction: a biomarker for COVID-19. Int Forum Allergy Rhinol. 2020;10:944-950.

4. Yan $\mathrm{CH}$, Faraji F, Prajapati DP, Boone CE, DeConde AS. Association of chemosensory dysfunction and COVID-19 in patients presenting with influenza-like symptoms. Int Forum Allergy Rhinol. 2020;10:806-813. 
5. Mao L, Jin H, Wang M, Hu Y, Chen S, He Q, et al. Neurologic manifestations of hospitalized patients with coronavirus disease 2019 in Wuhan, China. JAMA Neurol. 2020;77:1-9.

6. Russell B, Moss C, Rigg A, Hopkins C, Papa S, van Hemelrijck M. Anosmia and ageusia are emerging as symptoms in patients with $\mathrm{CO}$ VID-19: what does the current evidence say? Ecancermedicalscience. 2020;14:ed98.

7. Fox NS, Melka S. COVID-19 in pregnant women: case series from one large New York City obstetrical practice. Am J Perinatol. 2020; 37:1002-1004.

8. Nwankwo U, Fasunla AJ, Oladokun A, Nwaorgu OG, William SR. Comparison between olfactory function of pregnant women and non-pregnant women in reproductive age group in Ibadan, Nigeria. Niger $\mathrm{J}$ Clin Pract. 2017;20:610-615.

9. Soler ZM, Patel ZM, Turner JH, Holbrook EH. A primer on viral-associated olfactory loss in the era of COVID-19. Int Forum Allergy Rhinol. 2020;10:814-820
10. Xydakis MS, Dehgani-Mobaraki P, Holbrook EH, Geisthoff UW, Bauer C Hautefort C, et al. Smell and taste dysfunction in patients with COVID-19. Lancet Infect Dis. 2020;20:1015-1016.

11. Butowt R, Bilinska K. SARS-CoV-2: olfaction, brain infection, and the urgent need for clinical samples allowing earlier virus detection. ACS Chem Neurosci. 2020;11:1200-1203.

12. Joseph T, Auger SD, Peress L, Rack D, Cuzick J, Giovannoni G, et al. Screening performance of abbreviated versions of the UPSIT smell test. J Neurol. 2019;266:1897-1906.

13. Andrikopoulou M, Madden M, Wen T, Aubey JJ, Aziz A, Baptiste CD et al. Symptoms and critical illness among patients with coronavirus disease 2019 (COVID-19) infection. Obstet Gynecol. 2020:136:291-299.

14. Savasi VM, Parisi F, Patanè L, Ferrazzi E, Frigerio L, Pellegrino A, et al. Clinical findings and disease severity in hospitalized pregnant women with coronavirus disease 2019 (COVID-19). Obstet Gynecol. 2020;136:252-258 